\title{
Transnasal endoscopic and combined intra- extranasal approach for the surgical treatment of frontal sinus cerebrospinal fluid rhinorrhea
}

This article was published in the following Dove Press journal:

Therapeutics and Clinical Risk Management

9 June 2017

Number of times this article has been viewed

\section{Qintai Yang \\ Peng Li \\ Jiancong Huang \\ Weihao Wang \\ Shanyan Bian \\ Xuekun Huang \\ Xian Liu \\ Gehua Zhang}

Department of OtolaryngologyHead and Neck Surgery, The Third Affiliated Hospital of Sun Yat-sen University, Guangzhou, People's Republic of China
Correspondence: Qintai Yang; Peng Li Department of OtorhinolaryngologyHead and Neck Surgery, The Third Affiliated Hospital of Sun Yat-sen University, 600 Tianhe Road, Guangzhou 510630, People's Republic of China $\mathrm{Tel} / \mathrm{fax}+862085252239$

Email yang.qt@I63.com; Ip76@I63.net

\begin{abstract}
The aim of this study was to summarize and analyze the outcomes of frontal sinus cerebrospinal fluid rhinorrhea (FS-CSFR) treated by transnasal endoscopic and combined intra-extranasal approach. Clinical data on 20 cases of FS-CSFR patients from 2005 to 2013, with emphasis on the postoperative complications, clinical outcomes, and key technology involved in the combined intra-extranasal procedure, were retrospectively reviewed. Among the 20 cases, 12 were treated by combined intra-extranasal procedure; the other eight cases were initially treated by transnasal endoscopic approach alone, and five of them $(5 / 8,62.5 \%)$ were successfully treated and three failed. The three failed cases subsequently underwent combined intra-extranasal approach. A total of 15 cases, who received combined procedure, experienced fast recovery, had mild complications, and had no significant facial scars, and no CSFR recurrence was observed. Combined intra-extranasal approach offers advantages in not only overcoming the difficulty of insufficient exposure of defects during transnasal endoscopic procedure but also improving the success rate of repair.
\end{abstract}

Keywords: frontal sinus, cerebrospinal fluid rhinorrhea, endoscopy, otorhinolaryngologic surgical procedure, extracranial repair

\section{Introduction}

Cerebrospinal fluid rhinorrhea (CSFR) is the leakage of cerebrospinal fluid into the nasal cavity and paranasal sinuses through a damaged skull base. ${ }^{1}$ Frontal sinus CSFR (FS-CSFR) is often caused by the bone defect at the frontal posterior table, thus representing unique challenges for surgical repair. Surgical approaches used for dural repair include intracranial and extracranial approaches, endonasal approaches, and most recently endoscopic approaches. ${ }^{2}$ With improvements in endoscopic instrumentation, technology, and surgical expertise, endoscopic repair has truly become the gold standard in the management of anterior skull base defects, including FS-CSFR. Due to the postoperative large area of injury, the high incidence of complications, and the low cure rate, the intracranial approaches have now been gradually replaced by other approaches. ${ }^{3}$ However, each surgical approach has unique advantages and limitations. ${ }^{4}$ To investigate and maximize the advantages of transnasal endoscopic and "combined intra-extranasal" approach and improve the repair of the defect, we conducted a study on combined intra-extranasal approaches in 15 cases of FS-CSFR.

\section{Patients and methods}

\section{Clinical data collection}

Clinical data of all CSFR patients from 2005 to 2013 were retrieved from the health record and medical management system in the Third Affiliated Hospital of the Sun 
Yat-sen University, Guangzhou, People's Republic of China. All the selected patients were diagnosed as having CSFR based on their medical history, nasal endoscopic examination, high-resolution coronal and axial computed tomography (CT) and/or magnetic resonance imaging (MRI) scan, and the determination of glucose content of cerebrospinal fluid (CSF) subjects with CSFR outside the FS. Patients who had complication of hypoproteinemia or any other concomitant chronic organ disease were excluded from this study.

Case report forms were utilized to record the data on patient age, sex, etiology, location of the cerebrospinal fluid, surgery method, duration of the postoperative hospital stay, follow-up, and disease prognosis. Medical records included the following: endoscopic and imaging findings, surgery procedures, and postoperative courses.

There were 119 patients diagnosed as having CSFR in our hospital from 2005 to 2013. Of those patients, 20 (16.8\%) were diagnosed as having traumatic FS-CSFR. Patients' data were retrospectively analyzed and summarized (Table 1), and there were 19 males and one female (age range: 11-45 years). The lesions were mainly located in the posterior table of the FS with various extents of bony defects (maximum defect: $2.5 \mathrm{~cm} \times 3 \mathrm{~cm}$ ). Two cases were accompanied by meningoencephalocele. Three cases had pneumoencephalos (Figure 1). The time from trauma to the onset of FS-CSFR ranged from 2 days to 6 months. Each patient was informed of the purpose of the study and signed the written informed consent form including publishing of patient's data and images. This study was approved by the ethics review board of the Third Affiliated Hospital of Sun Yat-sen University.

\section{Conservative treatment}

According to the rules of our traditional treatment, patients were kept in bed with the head slightly elevated. An antibiotic (2.0 g ceftriaxone sodium) that may cross the blood-brain barrier was given by intravenous drip once a day, and $200 \mathrm{~mL}$ (25\%) of mannitol was given by intravenous drip four times a day to reduce the intracranial pressure. All efforts were made to reduce any straining or significant Valsalva maneuver, such as sneezing and coughing. Stool softeners were used. All the patients underwent surgeries after unsuccessful conservative measures for about half a month. However, two cases of the study population suffered from progressive pneumoencephalos and another one patient developed intracranial infection during the conservative treatment. We performed timely surgery and intensive antibiotic therapy instead of continuing conservative measures.

Table I Patient demographics, treatment, and outcomes with traumatic FS-CSFR

\begin{tabular}{|c|c|c|c|c|c|c|c|}
\hline $\begin{array}{l}\text { Patient } \\
\text { number }\end{array}$ & Sex & Age & Cause & Leakage site & Size & $\begin{array}{l}\text { Surgical } \\
\text { approach }\end{array}$ & $\begin{array}{l}\text { Treatment } \\
\text { success }\end{array}$ \\
\hline I & M & 36 & Motor vehicle accident & Medial plane of posterior table & $0.3 \times 0.4 \mathrm{~cm}$ & TER & Yes \\
\hline 2 & M & 17 & Motor vehicle accident & Medial plane of posterior table & $0.1 \times 0.3 \mathrm{~cm}$ & TER & Yes \\
\hline 3 & M & 33 & Motor vehicle accident & Medial plane of posterior table & $0.3 \times 0.3 \mathrm{~cm}$ & TER & Yes \\
\hline 4 & M & 19 & Motor vehicle accident & Medial plane of posterior table & $0.4 \times 0.5 \mathrm{~cm}$ & TER & Yes \\
\hline 5 & M & 22 & Motor vehicle accident & Medial plane of posterior table & $0.3 \times 0.6 \mathrm{~cm}$ & TER & Yes \\
\hline \multirow[t]{3}{*}{6} & M & 25 & Crash by door & Center of posterior table & $0.5 \times 1.0 \mathrm{~cm}$ & TER & No \\
\hline & & & & & & Second TER & No \\
\hline & & & & & & Combined approach & Yes \\
\hline \multirow[t]{2}{*}{7} & M & 13 & Fall down from $4 \mathrm{~m}$ high & Medial plane of posterior table & $0.5 \times 0.6 \mathrm{~cm}$ & TER & No \\
\hline & & & & & & Combined approach & Yes \\
\hline \multirow[t]{2}{*}{8} & M & 29 & Gun damage & Center roof of posterior table & $0.8 \times 1.5 \mathrm{~cm}$ & TER & No \\
\hline & & & & & & Combined approach & Yes \\
\hline 9 & M & 28 & Whirling emery wheel & Lateral roof of posterior table & $1.0 \times 1.8 \mathrm{~cm}$ & Combined approach & Yes \\
\hline 10 & M & II & Stab by sharp crabstick & Medial plane of posterior table & $0.8 \times 0.6 \mathrm{~cm}$ & Combined approach & Yes \\
\hline II & M & 13 & Fall down from the third floor & Lateral of posterior table & $1.5 \times 1.5 \mathrm{~cm}$ & Combined approach & Yes \\
\hline 12 & $\mathrm{~F}$ & 28 & Motor vehicle accident & Medial plane of posterior table & $0.8 \times 1.0 \mathrm{~cm}$ & Combined approach & Yes \\
\hline 13 & M & 45 & Motor vehicle accident & Lateral of posterior table & $0.1 \times 0.3 \mathrm{~cm}$ & Combined approach & Yes \\
\hline 14 & M & 21 & Motor vehicle accident & Lateral of posterior table & $0.9 \times 1.2 \mathrm{~cm}$ & Combined approach & Yes \\
\hline 15 & M & 43 & Motor vehicle accident & Center of posterior table & $0.2 \times 0.2 \mathrm{~cm}$ & Combined approach & Yes \\
\hline 16 & M & 17 & Motor vehicle accident & Center roof of posterior table & $0.5 \times 3.0 \mathrm{~cm}$ & Combined approach & Yes \\
\hline 17 & M & 32 & Motor vehicle accident & $\begin{array}{l}\text { Medial plane of posterior table } \\
\text { and frontal recess }\end{array}$ & $2.5 \times 3.0 \mathrm{~cm}$ & Combined approach & Yes \\
\hline 18 & M & 23 & Fall down from $5 \mathrm{~m}$ high & Center of posterior table & $1.2 \times 0.8 \mathrm{~cm}$ & Combined approach & Yes \\
\hline 19 & M & 34 & Motor vehicle accident & Lateral of posterior table & $0.3 \times 0.5 \mathrm{~cm}$ & Combined approach & Yes \\
\hline 20 & M & 38 & Motor vehicle accident & Lateral of posterior table & $1.5 \times 1.2 \mathrm{~cm}$ & Combined approach & Yes \\
\hline
\end{tabular}

Note: Combined approach: transnasal endoscopic combined with extranasal frontal open surgery.

Abbreviations: CSFR, cerebrospinal fluid rhinorrhea; FS, frontal sinus; TER, transnasal endoscopic repair. 


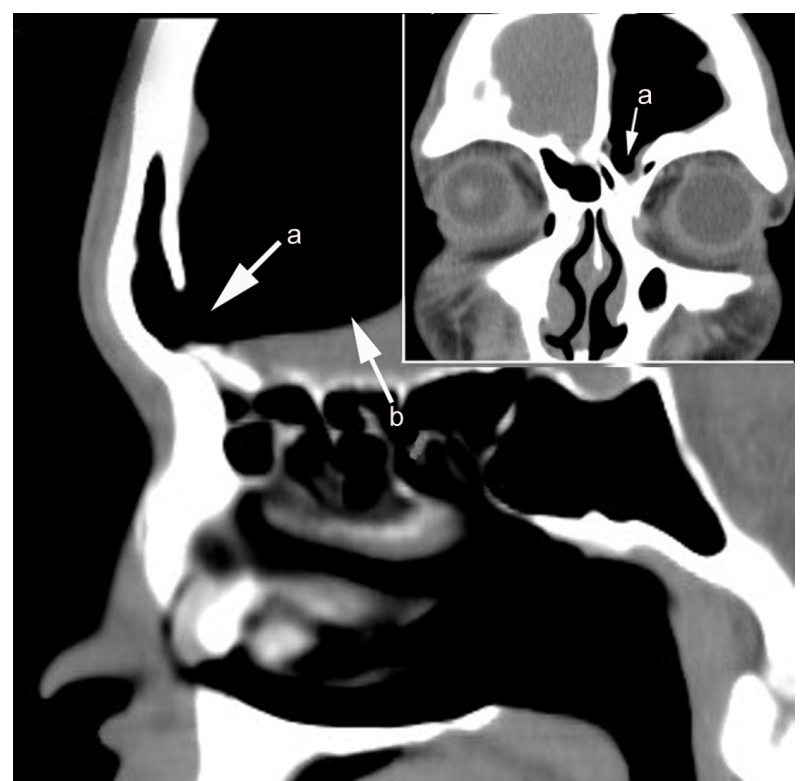

Figure I CT image in the sagittal and coronal plane: a, big defect of the posterior table of the FS; b, pneumoencephalos.

Abbreviations: CT, computed tomography; FS, frontal sinus.

\section{Surgery and perioperative procedures}

Surgical approach

In this study, we divided FS-CSFR into two types:

Type A: the defect was located in the medial plane of the lamina papyracea, and transnasal endoscopic frontal sinusotomy could provide adequate visualization and complete exposure of these defects. We classified eight of 20 patients as type A.

Type B: the defect was located in the medial plane of the lamina papyracea, but transnasal endoscopic frontal sinusotomy could not provide adequate visualization and complete exposure of these defects because of the small frontal ostium and poorly pneumatized agger nasi cell, or the defect was large (Figure 1) or located in the lateral to the plane of the lamina papyracea (Figures 2 and 3), that cannot be approached safely and successfully repaired endoscopically. It is difficult to reach satisfactory clinical outcomes for repairing those defects just by endoscopic technique. In our study, 15 of the 20 patients were classified as type $\mathrm{B}$, for those 15 patients, combined intra-extranasal approach was used.

\section{Transnasal endoscopic approach}

Uncinate process and ethmoid bulla were first removed by Messerklinger method. Wormald' $\mathrm{s}^{5}$ axillary flap technique or Draf's IIa or IIb or III procedure was utilized. ${ }^{6}$

\section{Combined intra-extranasal approach}

The procedure of combined intra-extranasal approach was completed by the following steps: transnasal endoscopic

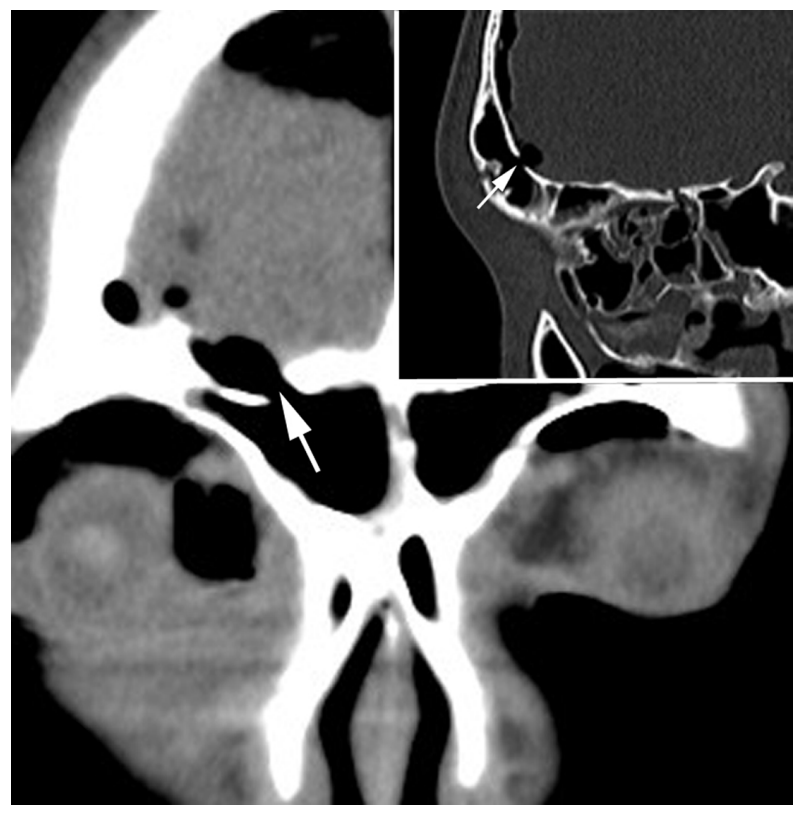

Figure $2 \mathrm{CT}$ image in the coronal and sagittal plane.

Note: The arrow indicates that the defect location was lateral to the plane of the lamina papyracea.

Abbreviation: $\mathrm{CT}$, computed tomography.

frontal sinusotomy was performed first, followed by extranasal FS open surgery (trephination):

1. Transnasal endoscopic approach: We conducted this procedure as the transnasal endoscopic approach that we have mentioned earlier.

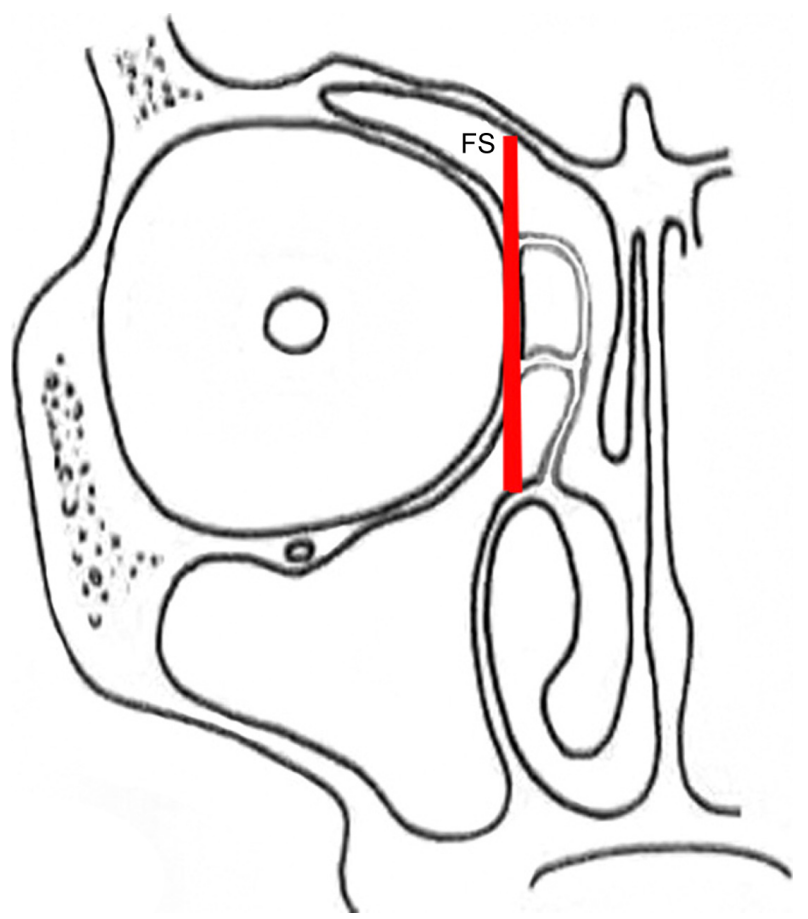

Figure 3 CSFR involved in the frontal posterior table can be classified into two types based on their relationship with the plane of the lamina papyracea (red line). Abbreviations: CSFR, cerebrospinal fluid rhinorrhea; FS, frontal sinus. 
2. Extranasal FS open surgery (trephination): After the defects were identified based on the imaging findings, the incision site inside the superciliary arch and the eyebrow with its opening at the FS was determined. An incision of $2-3 \mathrm{~cm}$ was made on the skin parallel to the eyebrow (the supratrochlear and supraorbital neurovascular bundles lie at the medial and lateral limits of the incision, Figure 4), a bone window comparable to the size of the defect was created by the drill bit and Kerrison rongeurs after exposure of the anterior table of the FS to gain access to the FS (Figure 5).

3. Repair of defect via the combined intra-extranasal approach: The FS outflow tract was opened by the combined intra-extranasal frontal open approaches, so that both the endoscopic and the surgical appliances could enter the FS from the two approaches (Figure 6). After exposure of the FS defect and identification of the exact site of the CSFR outflow, the defect bones, granulation, and some mucosa around the defects were cleared. A fresh grafting bed was made outside the dura mater, and the membrane covering bones at the edge of the dura mater defect was separated from that of the FS posterior table. The muscle-fascia flap obtained from the lateral aspect of the thigh was used as plugging and repair materials; muscle was grounded and stuffed between the dura mater and FS posterior table and

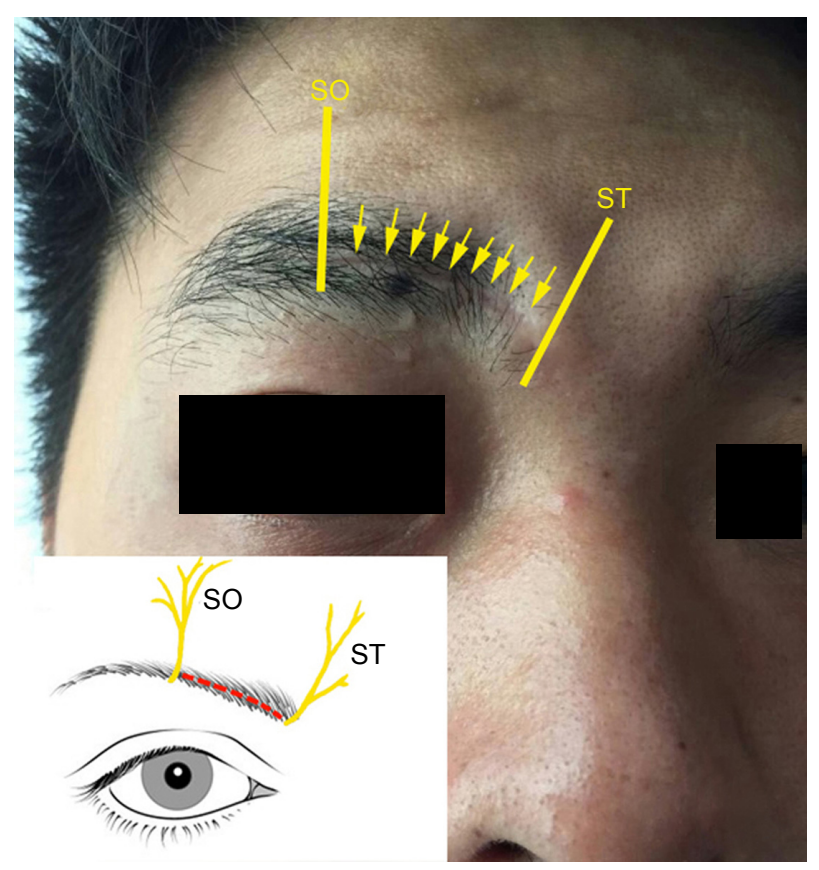

Figure 4 The incision that can be made inside the brow (yellow arrow and red line: intrabrow incision).

Note: The ST and SO neurovascular bundles lie at the medial and lateral limits of the incision.

Abbreviations: SO, supraorbital; ST, supratrochlear.

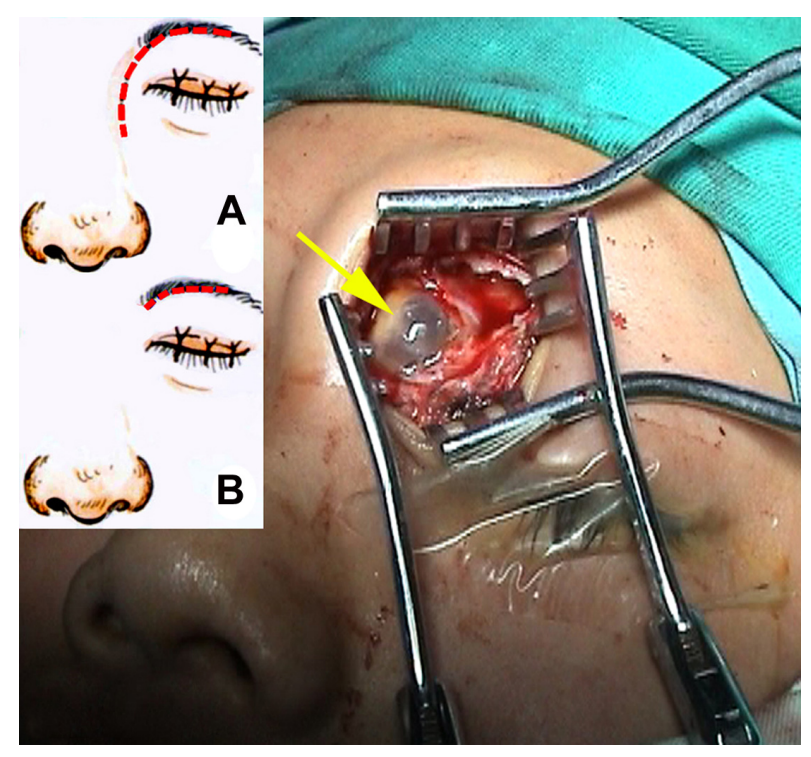

Figure 5 FS surgery through an extranasal approach.

Notes: The arrow indicates the defect of the posterior table of the FS and accompanied by meningoencephalocele (red line: A, bone window of the traditional extranasal frontal approach; B, bone window of the combined intra-extranasal approach).

Abbreviation: FS, frontal sinus.

outside the defect until the leakage of CSF stopped. Bone defect larger than $2 \mathrm{~cm}$ was fixed by titanium mesh plate which was then covered by fascia lata and glued with fibrin glue. The FS was further filled with gelatin sponge and iodoform gauze, and a silica gel drainage tube was placed to enable directional flow from the FS to the nasal cavity. Finally, the frontal incision was sutured.

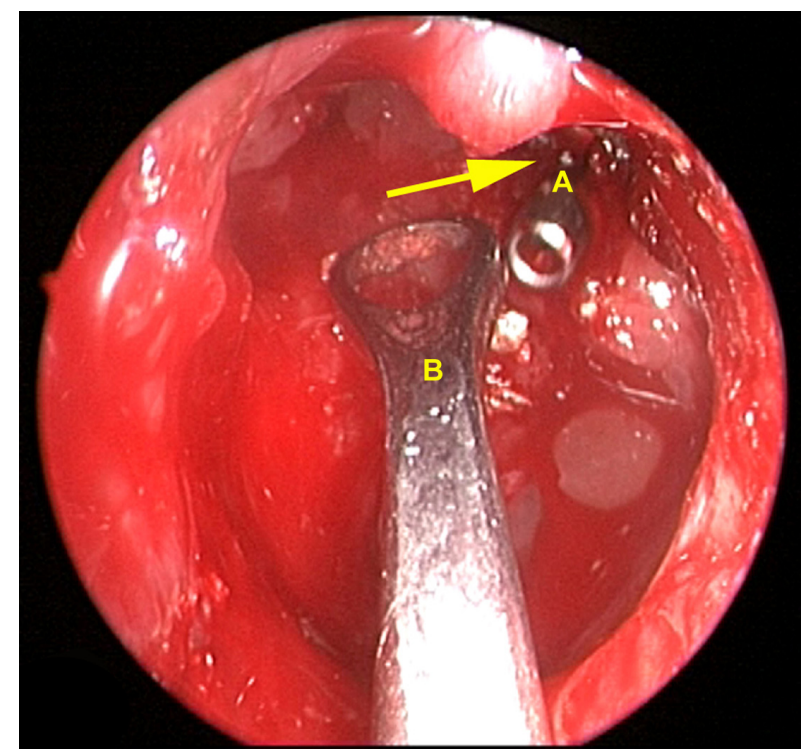

Figure 6 FS surgery through a combined intra-extranasal approach.

Notes: The arrow indicates the opening of the FS (A, transnasal approach; B, extranasal approach).

Abbreviation: FS, frontal sinus. 


\section{Perioperative cares}

During the surgery, antibiotic ( $2.0 \mathrm{~g}$ ceftriaxone sodium) and $200 \mathrm{~mL}(25 \%)$ of mannitol were infused within $30 \mathrm{~min}$ via intravenous drip. In the postoperative period, patients were treated according to the conservative measures that have been described earlier. The iodoform gauze and silica gel drainage tube were removed from the nasal cavity under the intranasal endoscope 10 days after surgery.

\section{Follow-up and evaluation}

Scheduled clinical visits, endoscopic examinations, and phone interviews were conducted to follow up the patients for the evaluation of surgical outcomes and complications. This group of patients had a follow-up period from 6 months to 8 years.

\section{Results}

Table 1 summarizes the clinical, treatment, outcome, and follow-up data of 20 patients with traumatic FS-CSFR. Among the 20 cases, 12 of them were successfully treated (no CSFR recurrence was found) by the combined intraextranasal approach at the first treatment. The remaining eight cases were initially treated by transnasal endoscopic approach alone, five $(62.5 \%)$ of them were successfully treated and three failed (Table 1 , patient 6 , patient 7 , and patient 8 ). One of the failed cases (Table 1, patient 6 ) was treated with a repeat endoscopic procedure but failed again. Then, the patient was successfully treated by the combined intra-extranasal approach. The other two failed cases (Table 1, patient 7 and patient 8 ) were successfully treated by the combined intraextranasal approach at the second attempt.

In this study, 15 cases (100\%) were successfully treated by the combination of intranasal endoscopy and extranasal FS open surgery. Bone defects in three cases (Table 1, patient 9, patient 11, and patient 17) were fixed with titanium mesh plate. Post-surgery recovery was fast, with no obvious facial scars. Drainage of FS remained unobstructed. No CSFR recurrence was found after 6 months to 10 years (mean 63 months) of follow-up (Figure 7). There were no serious postsurgical complications, except one case (Table 1, patient 17) of temporary numbness in the skin around the opening area at the forehead (Figure 8).

\section{Discussion}

Endoscopic surgical technique has been identified as the effective management of CSFR; the current study evaluated the clinical outcomes of transnasal endoscopic and combined intra-extranasal approach for the surgical treatment

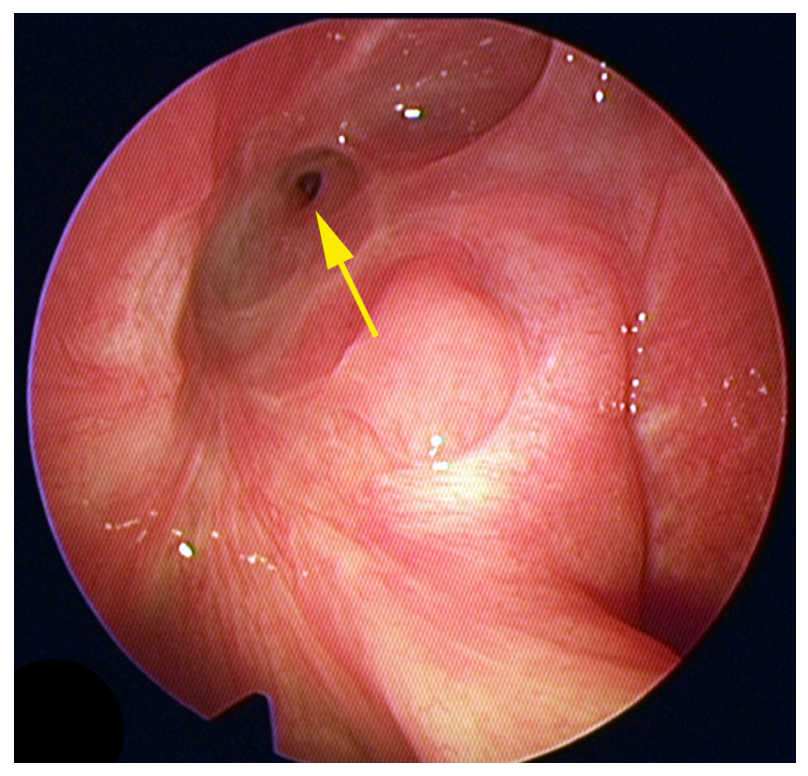

Figure 7 Postoperative transnasal endoscopic examination.

Notes: The FS-CSFR was successfully repaired, and the FS outflow tract was unobstructed. The arrow indicates the opening of the FS.

Abbreviations: CSFR, cerebrospinal fluid rhinorrhea; FS, frontal sinus.

of FS-CSFR. The current study found that all the FS-CSFR patients were successfully treated by the combined approach during their first surgery or subsequent resurgery. The combined intra-extranasal approach was shown to be a safe and effective treatment method for FS-CSFR and help lessen the postoperative complication with the mean follow-up of 63 months.

Since the first case of endoscopic repair of CSFR performed by Wigand, ${ }^{7}$ intranasal endoscopy has evolved to

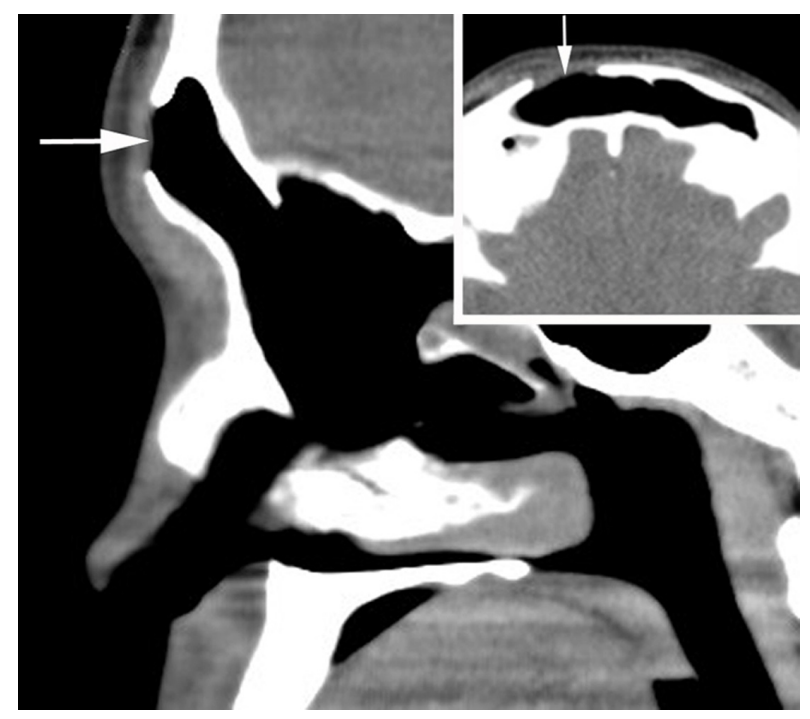

Figure 8 Postoperative CT image in the sagittal and axial plane. Note: The arrow indicates the bone window of the FS anterior table. Abbreviations: CT, computed tomography; FS, frontal sinus. 
become the first choice of treating CSFR of ethmoid and sphenoid sinuses. It has replaced other traditional approaches due to its safety, high efficacy, and limited injury. Recently, FS-CSFR leaks can be treated through an endoscopic endonasal approach in experienced surgical centers. However, in some situations where an endoscopic approach alone is insufficient, the combined intra-extranasal approach may be suitable, serving as an alternative to more invasive procedures. It is difficult to reach satisfactory clinical outcomes for repairing those defects just by endoscopic technique. In addition, an FS outflow tract may be blocked by the repair materials or postoperative adhesion, causing complications such as iatrogenic mucous cysts or FS inflammation; ${ }^{2}$ therefore, the cure rate of FS-CSFR is lower than CSFR at other locations. Transnasal endoscopic approach has been limited for FS-CSFR. ${ }^{8}$ Although the transnasal endoscopic modified Lothrop or Draf III procedure improved the treatment of FS-CSFR, its use has been limited due to its more severe surgical injury to the transnasal structure and more difficult surgery. ${ }^{9}$ Given the deficiency of transnasal endoscopic approach and the aggressive open surgery, some cases of FS-CSFR may benefit more from repair via the combined intra-extranasal approach. ${ }^{10,11}$

In this study, 15 patients underwent combined intraextranasal approach for FS-CSFR. Experience from these cases demonstrated that the combined approach could offer a more open and direct view to simplify and refine the repair of FS-CSFR leakage. It reduced the difficulty to locate and expose the defect, while increasing the cure rate. The opening of frontal recess by the endoscope maintained the structure of the FS and its original drainage pathway. In addition, the iodoform gauze and silica tube could be placed inside the sinus and easily taken out from the nasal cavity through the FS outflow tract, thus avoiding the secondary postoperative injury and iatrogenic complications.

The described combined approach allowed the simultaneous surgery via both the upper (eyebrow) and lower (nasal) approaches, which was particularly useful to repair the defects extending to the FS outflow tract. Interestingly, the combined approach could reduce the facial scar, because the operative incision was designed to be located inside the superciliary arch and covered by the eyebrow (Figure 4), which suggested that this combined approach was more likely to be accepted by patients compared to the single external open approach. ${ }^{12}$

Currently, the CSFR of FS and FS outflow tract is divided into three types based on anatomy: CSFR adjacent to the frontal recess, with direct involvement of the frontal recess, or located within the FS itself. ${ }^{2}$ Some researchers have proposed using individualized surgical approach to treat these different types of CSFR. ${ }^{13}$ Our experience indicates that CSFR adjacent to the frontal recess or inside the recess should be treated with the transnasal endoscopy since it will limit the complications to the minimum. ${ }^{11}$ On the other hand, CSFR involved in the frontal posterior table can be classified into two types based on their relationship to the plane of the lamina papyracea (Figure 6). Routine or transnasal endoscopic modified Lothrop or Draf III procedure may be preferable if the defect was located in the medial plane which was relatively easy to expose and operated by endoscope after enlargement of the FS ostium. Our study encompassed five patients in this type (type A). For those defects located in the lateral plane of the lamina papyracea or medial to the plane but difficult to expose during surgery (type B), it is recommended to use the combined approach. ${ }^{2,8,12}$

The current study showed that the combined intraextranasal approach achieved satisfactory outcomes in all 15 cases of FS-CSFR. However, if the patients have multiple fractures caused by head trauma, or a wide range of cranial damage and severe cranial base malformation, or CSFR accompanied by a high intracranial pressure that requires CSF shunt, or large area of frontal posterior table damage, the intracranial repair may still be necessary. If transnasal, extranasal, or the combined intra-extranasal approach cannot ensure that the postoperative FS outflow tract is unobstructed, patients can also be treated with traditional extracranial repair, such as osteoplastic flap and other open surgery repair with obliterated FS. ${ }^{13,14}$ Since the current study reviewed a limited number of cases with a uniform disease type, the indication and outcomes of the combined intra-extranasal approach still need to be confirmed with more cases and more diverse disease types.

\section{Conclusion}

All the patients with FS-CSFR were successfully managed by the combined intra-extranasal approach, and no CSFR recurrence was observed at the last clinical evaluation. Combined intra-extranasal approach offers advantages in not only overcoming the difficulty of insufficient exposure of defects during transnasal endoscopic procedure but also improving the success rate of repair. This technique is well tolerated by patients and may be easily incorporated into the rhinologist's practice.

\section{Acknowledgments}

Qintai Yang and Peng Li are first authors for this study. We thank all the participants of this study. 


\section{Disclosure}

The authors report no conflicts of interest in this work.

\section{References}

1. Oakley GM, Alt JA, Schlosser RJ, Harvey RJ, Orlandi RR. Diagnosis of cerebrospinal fluid rhinorrhea: an evidence-based review with recommendations. Int Forum Allergy Rhinol. 2016;6(1):8-16.

2. Illing EA, Woodworth BA. Management of frontal sinus cerebrospinal fluid leaks and encephaloceles. Otolaryngol Clin North Am. 2016;49(4): 1035-1050.

3. Yadav YR, Parihar V, Janakiram N, Pande S, Bajaj J, Namdev H. Endoscopic management of cerebrospinal fluid rhinorrhea. Asian J Neurosurg. 2016;11(3):183-193.

4. Martín-Martín C, Martínez-Capoccioni G, Serramito-García R, EspinosaRestrepo F. Surgical challenge: endoscopic repair of cerebrospinal fluid leak. BMC Res Notes. 2012;5:459.

5. Wormald PJ. The axillary flap approach to the frontal recess. Laryngoscope. 2002;112(3):494-499.

6. Shi JB, Chen FH, Fu QL, et al. Frontal sinus cerebrospinal fluid leaks: repair in 15 patients using an endoscopic surgical approach. ORL $J$ Otorhinolaryngol Relat Spec. 2010;72(1):56-62.

7. Wigand ME. Transnasal ethmoidectomy under endoscopical control. Rhinology. 1981;19(1):7-15.
8. Chaaban MR, Conger B, Riley KO, Woodworth BA. Transnasal endoscopic repair of posterior table fractures. Otolaryngol Head Neck Surg. 2012;147(6):1142-1147.

9. Sinha AK, Goyal S. Purely endoscopic pterional extradural approach: a novel technique for repair of cerebrospinal fluid rhinorrhea. J Neurosci Rural Pract. 2016;7(2):310-313.

10. Kirtane MV, Gautham K, Upadhyaya SR. Endoscopic CSF rhinorrhea closure: our experience in 267 cases. Otolaryngol Head Neck Surg. 2005; 132(2):208-212.

11. Roehm CE, Brown SM. Unilateral endoscopic approach for repair of frontal sinus cerebrospinal fluid leak. Skull Base. 2011;21(3): 139-146.

12. Patel AM, Vaughan WC. "Above and below" FESS: simple trephine with endoscopic sinus surgery. In: Kountakis SE, Senior BA, Draf W, editors. The Frontal Sinus. New York: Springer; 2005:212-219.

13. Woodworth BA, Schlosser RJ, Palmer JN. Endoscopic repair of frontal sinus cerebrospinal fluid leaks. J Laryngol Otol. 2005;119(9): 709-713.

14. Tosun F, Gonul E, Yetiser S, Gerek M. Analysis of different surgical approaches for the treatment of cerebrospinal fluid rhinorrhea. Minim Invasive Neurosurg. 2005;48(6):355-360.
Therapeutics and Clinical Risk Management

\section{Publish your work in this journal}

Therapeutics and Clinical Risk Management is an international, peerreviewed journal of clinical therapeutics and risk management, focusing on concise rapid reporting of clinical studies in all therapeutic areas, outcomes, safety, and programs for the effective, safe, and sustained use of medicines. This journal is indexed on PubMed Central, CAS,

\section{Dovepress}

EMBase, Scopus and the Elsevier Bibliographic databases. The manuscript management system is completely online and includes a very quick and fair peer-review system, which is all easy to use. Visit http://www.dovepress.com/testimonials.php to read real quotes from published authors.

Submit your manuscript here: http://www.dovepress.com/therapeutics-and-clinical-risk-management-journal 